\title{
Caracterización de membranas cerámicas de bajo coste para procesos avanzados de tratamientos de aguas
}

\author{
Olga Pérez Fernández ${ }^{1}$, Raquel Alcalá Borao ${ }^{1}$, Magda Lorente Ayza ${ }^{2}$, Enrique \\ Sánchez Vilches ${ }^{2}$, Sergio Mestre Beltrán ${ }^{2}$, Joaquín Coronas Ceresuela ${ }^{3}$, Miguel \\ Menéndez Sastre ${ }^{1}$
}

\author{
${ }^{1}$ Catálisis, Separaciones moleculares e Ingeniería de reactores (CREG) \\ Instituto de Investigación en Ingeniería de Aragón (I3A) \\ Universidad de Zaragoza, Mariano Esquillor s/n, 50018, Zaragoza, Spain. \\ Tel. +34-976762707, Fax +34-976762043, e-mail: olgapfer@unizar.es \\ ${ }^{2}$ Instituto de Tecnología Cerámica (ITC). Universidad Jaime I. Castellón. \\ ${ }^{3}$ Instituto de Nanociencia de Aragón (INA)
}

\begin{abstract}
En el presente trabajo se ha realizado la caracterización estructural de una línea de membranas de bajo coste, que se está desarrollando para su uso en tratamientos de aguas residuales, en reactores biológicos de membrana (MBR) y/o en tratamientos terciarios. Se comparan los resultados de tamaño de poro obtenidos en membranas cerámicas simétricas según dos métodos de caracterizacion: punto de burbuja y porosimetría de mercurio.
\end{abstract}

\section{Introducción}

Un reactor biológico de membrana (MBR) combina el proceso de degradación biológica con lodos activos y la separación directa líquido-sólido mediante una membrana de filtración(1). Los MBR presentan importantes ventajas como la reducción de espacio necesario, lo que implicaría una disminución del impacto ambiental y permiten obtener agua con concentraciones bajas de sólidos en suspensión. Uno de los mayores inconvenientes de los MBR es el ensuciamiento de membranas. A pesar del elevado coste de las membranas cerámicas, éstas presentan mayor hidrofilicidad que las poliméricas disminuyendo el ensuciamiento. También son más resistentes química, mecánica y térmicamente facilitando la limpieza mediante métodos físicos o químicos.

El método de punto de burbuja se utiliza para determinar tamaños de poro por encima de $50 \mathrm{~nm}$. Consiste en la introducción de un líquido en el interior de los poros de la membrana y se basa en la medida de la presión de aire que hay que aplicar para desplazar el líquido del interior de los poros.
La mínima presión a la que se observa paso de aire a través de la membrana corresponde al mayor tamaño de poro; este valor recibe el nombre de punto de burbuja (2)(3). La relación matemática entre la presión aplicada y el tamaño de poro viene dada por la ecuación de Washburn:

$$
\Delta P=\frac{4 \gamma \cos \varphi}{d}
$$

Donde $\Delta \mathrm{P}$ es la presión aplicada (bar), $\mathrm{d}_{\mathrm{p}}$ diámetro de poro $(\mu \mathrm{m}), \varphi$ el ángulo de contacto entre el fluido y las paredes del poro y $\gamma$ la tensión superficial del líquido.

El tamaño medio de poro $\left(\mathrm{d}_{50}\right)$ es el punto de corte de la representación de la línea del 50\% de flujo de aire $(\mathrm{mL} / \mathrm{min})$ que atraviesa la membrana seca frente a la presión aplicada con la curva equivalente para la membrana húmeda (ver figura 1).

La porosimetría de mercurio se basa en la ecuación de Washburn, al igual que el punto de burbuja, pero el fluido utilizado para rellenar los poros es mercurio. La diferencia entre ambos métodos es que el punto de burbuja mide el flujo de gas que atraviesa los poros, mientras que la porosimetría de mercurio mide el volumen de mercurio que penetra en los poros. Una limitación de la porosimetría de mercurio es que necesita trabajar a elevadas presiones, lo que puede provocar la rotura o deformación de la membrana (4). Otra limitación es que no diferencia entre los poros que determinan el flujo y los poros de soporte en membranas asimétricas.

El objetivo del presente estudio es comparar los resultados de tamaño medio de poro obtenidos 
mediante los métodos de punto de burbuja y porosimetría de mercurio de las membranas simétricas sintetizadas, para utilizarlas posteriormente en un MBR.

\section{Método Experimental}

Las membranas simétricas utilizadas fueron sintetizadas en el Instituto de Tecnología Cerámica (ITC), con materiales afines al sector cerámico tradicional de baldosas. Estas membranas fueron caracterizadas mediante la técnica de porosimetría de mercurio y punto de burbuja, llevadas a cabo por el $I T C$ y $C R E G$ respectivamente.

Para determinar el tamaño de poro mediante punto de burbuja es necesario realizar dos medidas de flujo de aire $(\mathrm{mL} / \mathrm{min})$. La primera se realiza con la membrana seca, midiendo el flujo de aire que atraviesa la membrana conforme aumenta la presión transmembrana (TMP). En la segunda medida se vuelve a medir el flujo de aire conforme aumenta la TMP con los poros de la membrana llenos de líquido y la membrana húmeda.

Se han estudiado 13 composiciones de soportes cerámicos diferentes y realizado 25 ensayos para comparar los valores de diámetro de poro medio obtenido mediante ambas técnicas.

\section{Resultados}

La representación de los resultados experimentales alcanzados con los métodos de porosimetría de mercurio y punto de burbuja muestra la existencia de una relación lineal entre ambos métodos con un $\mathrm{R}^{2}$ de 0,90 (ver figura 2).

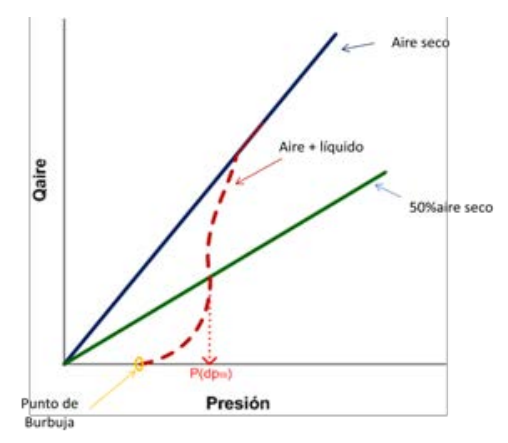

Figura 1. Representación fluido-presión

\section{Conclusiones}

Se ha estudiado la relación existente entre los dos métodos. La buena correlación existente entre ambos métodos se puede considerar una validación de la metodología empleada. Ello permitirá usar la técnica de punto de burbuja en el caso de membranas asimétricas, en las que la porosimetría de mercurio presenta una mayor dificultad en la determinación del tamaño de poro en la capa fina.

\section{Agradecimientos}

El presente trabajo muestra los resultados obtenidos en el proyecto WATERCER (IPT-2011-1069310000) que se ha realizado gracias al soporte económico del Ministerio de Ciencia e Innovación, en el marco del programa INNPACTO.

\section{Referencias}

1. P. Le-Clech, V. Chen, T. A. G. Fane, Fouling in membrane bioreactors used in wastewater treatment. J. Membr. Sci. 284, 17-53 (2006).

2. J. M. Benito, A. Conesa, M. A. Rodriguez, 2004. Ceramic membranes. Types, preparation methods and characterization. Boletin De La Sociedad Espanola De Ceramica Y Vidrio. 43, 829-842.

3. J. Yu, X. Hu, Y. Huang, 2010. A modification of the bubblepoint method to determine the pore-mouth size distribution of porous materials. Separation and Purification Technology. 70, 314-319.

4. A. Hernandez, P. Pradanos, J. I. Calvo, L. Palacio,1999. Ceramic membranes and their use in separation processes. Boletin De La Sociedad Espanola De Ceramica Y Vidrio. 38, 185-192.

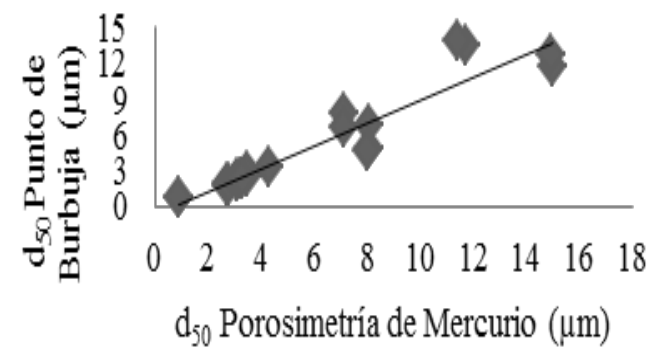

Figura 2. Comparación de los tamaños de poro medio medidos mediante la técnica de porosimetría de mercurio y punto de burbuja. 